\title{
Evaluación de las herramientas Web 2.0 en el periodismo digital: el caso de VilaWeb
}

\author{
Francisco HernándeZ SORIANO \\ Universidad de Lleida \\ paco.hernandez@udl.cat \\ Eduard CRISTÓBAL FRANSI \\ Universidad de Lleida \\ ecristobal@aegern.udl.cat
}

\begin{abstract}
Resumen:
Internet es un medio en continuo estudio donde sus principales resultados han ido asentando unas bases firmes para el establecimiento y promoción de los medios de comunicación a través de la Red. Es por ello que se ha llevado a cabo un análisis sobre VilaWeb por ser uno de los primeros portales de información aparecidos en España. Para ello, analizaremos su uso de las herramientas Web 2.0 en base al modelo ideado por Codina (2006) para después complementar los resultados con otros análisis de diversos autores.
\end{abstract}

Palabras clave: comunicación; Web 2.0; cibermedios; VilaWeb

\section{Evaluation of Web 2.0 tools in digital journalism: the case of Vilaweb}

\begin{abstract}
:
Internet is an ongoing study in which the matching results thereof have settled a firm basis for the establishment and promotion of the media through the Web. For this reason we realized an analysis on VilaWeb because is one of the first digital media appeared in Spain. So, we analyzed their use of Web 2.0 tools on the model devised by Codina (2006) to supplementing the results with other scholars' analysis.
\end{abstract}

Key Words: communication; Web 2.0; digital media; VilaWeb

Referencia normalizada:

Hernández Soriano, F. y Cristóbal Fransi, E. (2014): Evaluación de las herramientas web 2.0 en el periodismo digital: el caso de VilaWeb. Historia y Comunicación Social. Vol. 19. Núm. Especial Enero. Págs. 79-91.

Sumario: 1. Introducción: VilaWeb como cibermedio. 2. Análisis básico de las herramientas Web 2.0. 3. Conclusiones. 4. Bibliografía. 


\section{Introducción: VilaWeb como cibermedio}

Vicent Partal define VilaWeb como: "la puerta de entrada del lector a la globalidad. VilaWeb lo hemos definido a veces diciendo que es un medio glocall. Es decir, que presenta una mirada local sobre la globalidad. Nos hemos planteado ser los continuadores de la tradición de medios de comunicación de proximidad en nuestro país, con la prensa comarcal y local, y las radios y las televisiones locales y comarcales...". (Partal, 2007. Pág. 64).

Ahora bien, de forma más técnica y centrándonos en una extensa revisión de la literatura; los trabajos recogidos por Salaverría (2003 y 2005), la definición propuesta por López et al. (2005), la especificación de tipificaciones de Palacios y Díaz (2009), la clasificación elaborada por Orihuela (2005), Meso (2007) y Masip (2008) podríamos calificar VilaWeb como: un Cibermedio nativo, de contenido general y local, de actualización constante, que contempla prácticamente todos los modelos de comunicación, con un grado de adecuación de nivel 5 y cuya finalidad de contenidos es: informativa, comunicativa, y de intermediación y servicios.

\section{Análisis básico de las herramientas web 2.0}

Para llevar a cabo el presente análisis nos hemos decantado por el modelo de análisis elaborado por Codina (2006), que más recientemente ha ampliado en 2012 pero sin entrar en el ámbito estrictamente periodístico y centrándonos únicamente en el uso de la herramientas Web 2.0. El modelo de Codina (2006) distingue entre dos tipos de indicadores: generales y específicos. Los primeros valoran la totalidad de la web independientemente de su género, tema, etc., mientras que los específicos valoran los elementos característicos propios de la página diferenciándolos entre internos (los que la página debería tener) y externos (aquellos que permiten observar el impacto de la web social en la prensa digital).

El análisis que se va a llevar a cabo a continuación comprendió un periodo de observación durante los meses de mayo y septiembre de 2013. Asimismo, los resultados se compararon con un estudio semejante realizado en la Universidad de Lleida durante el año 2010 y, de este modo, podremos ver si el portal de información se ha adaptado a los cambios producidos en Internet en los últimos años.

\subsection{Accesibilidad de VilaWeb}

Tanto Díaz (2005) como Rodríguez-Martínez et al. (2010) confieren gran importancia a este apartado puesto que se trata de comprobar "el nivel de adecuación de sus

El término Glocal, incluido en el Diccionario Oxford de las Nuevas Palabras (1991:134), mezcla los términos global y local para determinar el hecho de que se adaptan las visiones globales a las condiciones locales. 
contenidos a los usuarios con características especiales, como deficiencias visuales, auditivas, o motrices, o que utilicen tecnologías de capacidad limitada..." (Rodríguez-Martínez et al., 2010. Pág. 37).

Existen tres niveles de adecuación: A, AA y AAA, a los cuales se les ha asociado a su vez un nivel de prioridad en función del impacto en la web de cada uno de ellos:

- A (Prioridad 1): son aquellos requisitos que "tiene" que satisfacer VilaWeb para que los usuarios con necesidades especiales puedan acceder a la información.

- AA (Prioridad 2): aquellos que "debe" cumplir para facilitar su accesibilidad.

- AAA (Prioridad 3): aquellos requisitos que "puede" cumplir para optimizar la accesibilidad de sus usuarios.

Díaz (2005) y Rodríguez-Martínez (2010) dejan patente que existen varias aplicaciones y páginas web online que pueden llevar a cabo el análisis del nivel de accesibilidad de la web: Ocawa, Hera y Taw (Test de Accesibilidad a la Web). Debido a la reciente actualización de su programario se llevó a cabo el análisis de VilaWeb mediante el T.A.W. [http://www.tawdis.net/]. Los resultados obtenidos se pueden observar en la siguiente tabla:

Tabla 1: Nivel de accesibilidad de la página principal de VilaWeb realizado el 17 de septiembre de 2013

\begin{tabular}{|l|l|l|l|l|}
\hline \multirow{2}{*}{ VilaWeb } & \multicolumn{4}{|l|}{ Número de errores de Accesibilidad (mediante el test Taw) } \\
\cline { 2 - 5 } & Prioridad 1 & Prioridad 2 & Prioridad 3 & Total \\
\hline 2010 & 4 & 46 & 0 & 50 \\
\hline 2013 & 8 & 55 & 0 & 63 \\
\hline
\end{tabular}

VilaWeb muestra, en resumen, un bajo número de errores en la codificación para facilitar el acceso a sus noticias, sobre todo si lo comparamos con otros diarios como Elmundo que sobrepasan los 200 errores. Así pues, a pesar del incremento de errores en los últimos tres años también, hay que advertir que la web ha incrementados sus servicios y secciones.

\subsection{Visibilidad y popularidad de VilaWeb}

En este apartado, el modelo de análisis se sirve de tres parámetros diferentes:

- PageRank de Google: se trata de saber la trascendencia que tiene para Google la página web en cuestión.

- Traffic Rank de Alexa: se encarga de posicionar la web en referencia a un ranking nacional e internacional de las páginas más visitadas, así como un análisis en cuanto a la tipología de los usuarios.

- En un primer momento Codina (2006) usó Yahoo Site Explorer (YSE) para evidenciar la visibilidad o popularidad de los Cibermedios estudiados en 
función de los enlaces recibidos y las páginas indexadas. Actualmente YSE ya no existe, aunque sin embargo han aparecido webs de igual capacidad como Open Site Explorer.

De ese modo, $\mathrm{y}$ en un primer resumen, el resultado ha sido el siguiente:

Tabla 2: Nivel de visibilidad y popularidad de VilaWeb realizado el 17 de septiembre de 2013

\begin{tabular}{|l|l|l|l|}
\hline Vilaweb & $\begin{array}{l}\text { Page Rank } \\
\text { (google) }\end{array}$ & $\begin{array}{l}\text { Traffic Rank (Alexa) } \\
\text { Nacional e Internacional }\end{array}$ & $\begin{array}{l}\text { Open Site Explorer } \\
\text { (OSE) }\end{array}$ \\
\hline 2010 & $6 / 10$ & $935 / 52.142$ & - \\
\hline 2013 & $6 / 10$ & $486 / 21.913$ & $78 / 100$ \\
\hline
\end{tabular}

El resultado de PageRank indica que VilaWeb, como diario electrónico, tiene una posibilidad de 6 entre 10 de aparecer en búsquedas a través de Google. Respecto al año 2010 el resultado es el mismo, dato que no ha mejorado a diferencia de otros diarios como LaVanguardia.com que han pasado de un 7/10 a un 8/10. Sin embargo, según $O S E$ predice una posibilidad de 78 sobre 100 de que la web aparezca en una consulta realizada a través de motores de búsqueda.

En términos numéricos a nivel nacional e internacional, VilaWeb ocupa el puesto 486 de las webs más visitadas en España y el 21.913 del mundo. Otros diarios como Elmundo y ElPaís ocupan los puestos 11 y 12 respectivamente. Pese a ello, ha habido una notable mejoría desde 2010 subiendo casi quinientos puestos en España. Por otro lado, dichos resultados coinciden con las estadísticas de la OJD (1997-2013) en referencia al tiempo medio diario de visita en VilaWeb por cada usuario, situándolo entre una media de 2 y 3 minutos. Destaca el hecho de que un alto porcentaje de los usuarios que acceden a VilaWeb provienen de Facebook y de Google.

Continuando con la visibilidad, pasamos a estudiar qué formas o sistemas contiene el portal de información para que los usuarios puedan acceder a la información. Rodríguez-Martínez et al. (2010) dividen este análisis en cuatro tipos, cuyos resultados tanto para 2010 como para 2013 fueron los siguientes:

Tabla 3: Puntos de acceso a la información en VilaWeb en un análisis llevado a cabo el 12 de mayo y el 17 de septiembre de 2013.

\begin{tabular}{|l|l|l|l|l|}
\hline Medio & $\begin{array}{l}\text { Búsqueda } \\
\text { simple }\end{array}$ & $\begin{array}{l}\text { Búsqueda } \\
\text { avanzada }\end{array}$ & Hemeroteca & Mapa de la Web \\
\hline VilaWeb & Sí & No & Sí & Sí \\
\hline
\end{tabular}

Búsqueda simple: VilaWeb cuenta con un buscador que permite acceder a la información de la web y de otros motores de búsqueda. Lo importante del buscador es que se encuentra disponible en todas las secciones e incluso en las ediciones locales. 
Búsqueda avanzada: En ningún momento se puede referenciar la búsqueda por sección o por autor, sobre todo porque el nombre de éstos últimos únicamente aparece en los artículos de opinión pero no en las noticias diarias.

En lo referente al apartado de Hemeroteca, el portal cuenta con una hemeroteca eficiente que permite realizar la consulta por título y fecha, siempre y cuando sea a partir del 28 de noviembre de 1998.

Mapa Web: VilaWeb cuenta con un mapa web que estructura la información tal y como aparece en la portada. Sin embargo, en el momento del análisis del presente trabajo el mapa estaba desactivado a pesar de constar en dos partes de la página: en la propia portada y al final de la misma.

Pasando a indicadores específicos internos analizamos en primer lugar hasta qué punto puede el lector obtener información de cualquier información que aparezca publicada en VilaWeb. De ese modo se observaron los siguientes resultados

Tabla 4a: Profundización en la información, análisis llevado a cabo el 12 de mayo y el 17 de septiembre de 2013.

\begin{tabular}{|l|l|l|l|l|}
\hline VilaWeb & Hora Actual & Autor Noticia & Enlace Sección & Fotog. Infog. \\
\hline 2010 & Sí & No & No & Sí \\
\hline 2013 & Sí & No & No & Sí \\
\hline
\end{tabular}

Tabla 4b: Profundización en la información, análisis llevado a cabo el 12 de mayo y el 17 de septiembre de 2013.

\begin{tabular}{|l|l|l|l|l|}
\hline VilaWeb & Audio Vídeo & Enlace Hemerot. & Enlace Externo & Artículo Autor \\
\hline 2010 & Sí & No & Sí & No \\
\hline 2013 & Sí & No & Sí & No \\
\hline
\end{tabular}

- Hora actual: ¿Aparece la hora de la última actualización? VilaWeb incluye la hora y el día en que la noticia aparece en todas y cada una de sus secciones.

- Autor Noticia: ¿Se identifica el autor de la noticia? Negativo, únicamente en ocasiones y si se trata de la Agencia Catalana de Noticias (ACN).

- Enlace sección: Cuando se accede al texto de una noticia ¿Hay enlaces a artículos de la misma sección? Negativo. Sin embargo es curioso el hecho de que incluya enlaces a otros diarios como Le Monde con la misma noticia referida o a vídeos de VilaWebTV relacionados.

- Enlace hemeroteca: ¿Hay enlaces a artículos de la hemeroteca digital? La única forma de acceder a los artículos de la hemeroteca es a través de la sección de la misma. 
- Enlace externo: ¿Se facilitan enlaces a artículos o informaciones externas al propio diario para contextualizar las noticias? sí, independientemente de la temática o sección de la que provenga la información.

- Artículo Autor: ¿Es posible acceder a los artículos de un autor concreto? Únicamente permite la opción de búsqueda por autores cuando se trata de artículos de opinión en la sección Mail Obert.

- Fotografía/Infografía: ¿Se emplea contenido gráfico para cubrir o ampliar la cobertura de las noticias? La práctica totalidad de las noticias observadas contaban con apoyo visual mediante fotografías y/o vídeo.

- Audio/Vídeo: ¿Se emplea contenido en formato de audio o vídeo para cubriro ampliar la cobertura de las noticias? Sí, e incluso cuenta con un canal propio en diversas plataformas y en la propia web.

\subsection{Herramientas de interacción}

$\mathrm{Al}$ igual que en el indicador anterior se realizan una serie de preguntas en base al nivel de interacción entre el lector y VilaWeb. Los resultados fueron los siguientes:

Tabla 5a: Herramientas de Interacción, análisis realizado el 12 de mayo y el 17 de septiembre de 2013.

\begin{tabular}{|l|l|l|l|l|l|}
\hline VilaWeb & Blog & Blog Usuarios & Noticias + Leídas & Coment. & Compartir \\
\hline 2010 & $\mathrm{Si}$ & $\mathrm{Sí}$ & No & No & Sí \\
\hline 2013 & $\mathrm{Si}$ & $\mathrm{Sí}$ & No & No & Sí \\
\hline
\end{tabular}

Tabla 5b: Herramientas de Interacción (continuación), análisis realizado el 12 de mayo y el 17 de septiembre de 2013.

\begin{tabular}{|l|l|l|l|l|}
\hline VilaWeb & Contactar Autor & Contrib. De los lectores & Foro & Chat \\
\hline 2010 & No & No & Sí & Sí \\
\hline 2013 & No & Sí & Sí & Sí \\
\hline
\end{tabular}

- Blog: ¿Dispone el diario de un blog? El diario cuenta con varios blogs especializados realizados por varios autores.

- Blog usuarios: ¿Ofrece la posibilidad de crear un blog a sus usuarios dentro de su propio dominio? Afirmativo, e incluso cuenta con un apartado para los más destacados del día.

- Comentarios: ¿Se pueden hacer comentarios a las noticias publicadas? No, sin embargo sí que se permite comentar los videos en YouTube. Durante el estudio de 2010 se escogieron de muestra, al azar, 110 vídeos (de entre todas las secciones) de los 1.100 (un 10\% del total aproximadamente) de los que disponía VilaWeb en YouTube. El resultado fue que, de estos 110, 72 tenían habilitada la opción de ser comentados, 5 no estaban disponibles y el resto (33) 
no podían ser comentados por los usuarios que visualizaran el vídeo. Ahora bien, al analizar los vídeos por antigüedad nos percatamos de que cuanto más reciente era el vídeo menos posibilidad existía que pudiera ser comentado, independientemente de la tipología y el contenido del vídeo. Actualmente, de una muestra escogida de 160 vídeos, únicamente 20 no podían visualizarse, perteneciendo la mayoría a secciones concretas que no formaban parte de noticias de última hora a diferencia de 2010.

- Compartir: ¿Dispone de herramientas Web 2.0 para compartir contenidos? Sí, las noticias pueden ser enviadas directamente a través de Facebook y Twitter.

- Contactar autor: ¿Permite a los lectores contactar vía correo electrónico con el autor de una noticia? Al no estar identificados éstos con las noticias no se puede llevar a cabo dicho contacto.

- Contribuciones de los lectores: ¿Acepta contribuciones de los lectores? En 2010 no se observó ningún caso en concreto. Sin embargo, en 2013, se incorpora un servicio que informa a los lectores de los temas que se van a tratar al día siguiente para que así puedan colaborar en la medida de lo posible.

- Foros de discusión: ¿Dispone de foros de discusión? Afirmativo, cuenta con una sección únicamente dedicada a los debates que se generan a raíz de las noticias que publican $u$ otros temas de actualidad

- Chat: ¿Tiene chats para sus usuarios? Si en algo se diferenció VilaWeb en un primer momento desde 1997 fue en contar con una sección de chats. Dispone tanto de canales propios como de otros en diferentes servidores.

- Noticias más leídas: ¿Posee una sección con las noticias más leídas? Hasta la fecha no existe en VilaWeb ningún espacio, sección o servicio que indique cuales han sido las noticias más leídas del día.

\subsection{Personalización de la información}

En este punto se trata justamente de ver hasta qué punto está preparada VilaWeb para reaccionar diariamente frente a la demanda de lectores que deseen información o comunicarse a través de su página web desde cualquier parte, y en cualquier momento.

Tabla 6: Personalización de la información de VilaWeb el 12 de mayo y el 17 de septiembre de 2013.

\begin{tabular}{|l|l|l|l|l|l|l|}
\hline VilaWeb & Alertas & RSS & Correo & Móvil & Registro & Personalizar \\
\hline 2010 & Sí & Sí & Sí & Sí & Sí & No \\
\hline 2013 & Sí & Sí & Sí & Sí & Sí & No \\
\hline
\end{tabular}

- Alertas: ¿Posee un servicio de alertas? Únicamente el Boletín Informativo que se manda por correo electrónico. 
- RSS: ¿Dispone de canales de contenido sindicado? VilaWeb ofrece la posibilidad de suscribirse a canales RSS para Google, Blogline y otras aplicaciones.

- Correo: ¿Es posible el envío de noticias vía correo electrónico? Previa suscripción, envía a sus usuarios un boletín diario con las noticias más actuales.

- Móvil: ¿Facilita el envío de noticias a teléfonos móviles? Afirmativo, incluso se han adaptado a las nuevas tecnologías de smartphones.

- Registro: ¿Ofrece a sus usuarios la opción de registrarse? Mediante MesVilaWeb, los lectores pueden recibir más información o servicios dependiendo de la cuota que suscriban.

- Personalizar: ¿Cuenta con aplicaciones que permitan a sus usuarios personalizar los contenidos del diario? Negativo, la única aplicación a través de la cual el lector puede personalizar el contenido es su espacio de blog personal.

2.5. Presencia de VilaWeb en las redes sociales

El siguiente de los indicadores específicos pretende determinar hasta qué punto VilaWeb cuenta con las plataformas de redes sociales para así difundir información más allá de la página web y captar lectores a través de ellas.

Tabla 7: Presencia de VilaWeb en redes sociales el 17 de septiembre de 2013

\begin{tabular}{|l|l|l|l|l|l|}
\hline VilaWeb & Facebook & Tuenti & Twitter & Myspace & LinkedIn \\
\hline 2010 & Sí & No & No & No & No \\
\hline 2013 & Sí & No & Sí & No & No \\
\hline
\end{tabular}

Se ha de tener en cuenta el dato de Alexa, según el cual la gran mayoría de los usuarios de VilaWeb procedían directamente de Facebook.

\subsection{Presencia de VilaWeb en plataformas web 2.0}

Las plataformas Web 2.0 se han especializado en el alojamiento y difusión de contenidos y, gracias a ello, los medios de comunicación han podido proporcionar a sus usuarios no sólo nuevos canales de comunicación sino también diversidad de opciones a la hora de poder acceder a la información. Se han escogido tres herramientas basándonos, según Rodríguez-Martínez et al. (2010), en: sitios web que disfrutan de mayor popularidad entre los usuarios, y sitios web que se emplean de forma más habitual por los medios de comunicación analizados.

Tabla 8: Presencia de VilaWeb en plataformas Web 2.0 el 12 de mayo y el 17 de sept. de 2013

\begin{tabular}{|l|l|l|l|}
\hline VilaWeb & Youtube & Flickr & Twitter \\
\hline 2010 & Sí & No & No \\
\hline 2013 & Sí & No & Sí \\
\hline
\end{tabular}


Una vez más recalcar el hecho de que finalmente dispone de cuenta en Twitter, algo que hace tres años no sucedía a pesar de estar incluida entonces en el mapa del portal. Sucede todo lo contrario con YouTube, donde tiene alojados más de 1.600 vídeos, 500 más que en 2010.

\subsection{Análisis complementarios}

Tal y como comentamos anteriormente, López et al. (2005) realizaron una propuesta de tipificación de Cibermedios en base al "grado de adecuación" de estos. En resumen, se pretendía clasificar un cibermedio en base al grado de aprovechamiento que éste hace las posibilidades que Internet le ofrece. Para ello crearon cuatro grandes indicadores en torno a los cuales se desarrollaba un test para confirmar dicho grado de adecuación:

- Hipertextualidad: trataría de definir el grado en el que un lector puede llegar a profundizar en la noticia a través de los hipervínculos y la navegación en la página web.

- Multimedialidad: valora el apoyo multimedia que la noticia o información reciben sin duplicarla.

- Interactividad: En este apartado, López et al (2005) no hacen hincapié únicamente en el grado en que el lector puede comentar o ser partícipe de la noticia sino que, además, incluyen como parte del indicador que el internauta tenga facilidad para disponer de los contenidos en la página web.

- Frecuencia de actualización: se basa en la "continuidad y profundidad informativas", así como en la rapidez con la que ésta se actualiza durante el día.

Estos cinco indicadores pueden puntuarse del 1 al 5, siendo el 5 la mayor frecuencia o intensidad con la que se identifica al indicador. La suma de las puntuaciones que ofrezca cada indicador determinará el "grado de adecuación" del cibermedio, siendo dichos grados clasificados en 5 categorías tal y como proponen López et al. (2005):

- Cibermedios de nivel 1: los cuatro indicadores suman entre 0 y 4

- Cibermedios de nivel 2: los indicadores suman entre 5 y 8

- Cibermedios de nivel 3: los indicadores suman entre 9 y 12

- Cibermedios de nivel 4: los indicadores suman entre 13 y 16

- Cibermedios de nivel 5: los cuatro indicadores suman entre 17 y 20

Los resultados que se van a presentar a continuación provienen de los mismos datos en que nos basamos para trabajar con el modelo de Codina (2006). 
Figura 1. Test sobre el grado de adecuación de VilaWeb como Cibermedio. López et al. (2005) y elaboración propia

- Nombre del cibermedio: VilaWeb

- Propiedades:

- Grado de adecuación de la Hipertextualidad

$0|1| 2|3| \mathbf{4} \mid 5$

- Grado de adecuación de la Multimediadlidad

$0|1| 2|3| 4 \mid 5$

- Grado de adecuación de la Interactividad

$0|1| 2|\mathbf{3}| 4 \mid 5$

- Grado de adecuación de la Frec. de Actualización $0|1| 2|3| 4 \mid \mathbf{5}$

- Total: $0|1| 2|3| 4|5| 6|7| 8|9| 10|11| 12|13| 14|15| 16 \mid$ 17 | 18 | 19 | 20

- Cibermedio de nivel: $0|1| 2|3| 4 \mid 5$

Por otro lado, en la "Actualización del modelo de portal periodístico de prensa española” de López y Pastor (2010), hacían hincapié en que en los últimos años habían aparecido numerosas novedades teóricas, prácticas y tecnológicas suficientes como para que los medios de comunicación en la red se pudieran diferenciar en dos estadios de evolución: estándar y avanzado. En base a ello, y los resultados previos obtenidos, podríamos decir que VilaWeb es un Cibermedio en un estadio avanzado.

\section{Conclusiones}

A través del presente análisis se ha podido observar, tanto a través de su estructura y composición como de su historia, que VilaWeb ha sido siempre pionera en la implantación de servicios y herramientas Web 2.0. Su afán por paliar el número de usuarios reducido a causa del idioma utilizado, su estilo editorial, que no cuenta con edición impresa, y el hecho de primar las noticias locales sobre las nacionales e internacionales, la llevan a intentar abarcar todos los medios comunicativos y fórmulas necesarias para llegar al mayor número de lectores posible.

En definitiva, se podría resumir el presente trabajo destacando los puntos fuertes y las debilidades de VilaWeb como diario electrónico.

En cuanto a los puntos fuertes:

- Audiencia. A pesar de ir dirigido a las zonas de habla catalana, y aunque no se encuentra entre los diarios más leídos de nuestro país, se ha podido observar que, a excepción de 2010 y 2011, ha habido un incremento continuo de su audiencia.

- Accesibilidad a la web y a la información: según el análisis realizado por el TAW, VilaWeb muestra que la fiabilidad y las posibilidades de acceder en un primer momento a las noticias de la página web son más altas que en otros diarios digitales. 
- Multimedialidad. Quizá el punto más fuerte con el que cuenta VilaWeb, que se ha sabido rodear no sólo de herramientas Web 2.0 sino también de expandirse a otras páginas como YouTube para ampliar la captación de usuarios.

- Hipertextualidad: Como portal de información que es, todas las noticias son complementadas con información acerca de organizaciones, personas jurídicas, físicas, entidades, etc. que en ese momento estén relacionadas con la noticia.

- Actualización: VilaWeb se actualiza durante todo el día, independientemente de la hora.

- Personalización de la información: Desde un primer momento VilaWeb ha tenido en cuenta la sindicación de contenidos y que el lector tenga facilidades a la hora de recibirlos. De ahí que fuera el primer diario digital en contar con sistema WAP de aviso a teléfonos móviles y PDAs.

Ahora bien, por otro lado muestra una serie de deficiencias y debilidades que sitúan a VilaWeb en clara desventaja respecto a otros diarios electrónicos en línea:

- Interactividad: a pesar de una mejor en cuanto a la elaboración de contenidos, el internauta no puede comentar las noticias a tiempo real, a diferencia de otros diarios digitales.

- Profundización de la información: los internautas tampoco pueden contactar o conocer quién es el autor de la noticia y mucho menos realizar búsquedas de los artículos en base a esa característica ya que no hay referencia alguna en ninguna de las publicaciones.

- Servicios de pago como los blogs o el correo electrónico deben ser mejorados ya que, cada vez menos, los usuarios de Internet no están dispuestos a pagar por algo de lo que pueden disponer de forma gratuita.

En resumen, VilaWeb no puede (ni debe) descartar ningún elemento, posibilidad o servicio que le permita una mejor captación de los lectores y un mejor posicionamiento en cuanto al resto de diarios digitales. Además, no debe basar su estrategia competitiva únicamente en la diferenciación de su diseño y contenido, sino también en poder ofrecer a sus usuarios un sistema informativo y un portal de comunicaciones ágil, cercano, ameno y fiable. Sobre todo, y teniendo en cuenta, que Internet ofrece un gran número de herramientas y aplicaciones de forma gratuita o bajo coste que facilitan la consecución de dicho objetivo y una interactividad por parte del internauta que puede mejorar su posicionamiento como diario electrónico.

\section{Bibliografía}

CODINA, L. (2006). Metodología de Análisis y Evaluación de Recursos Digitales en Línea. Trabajo que forma parte del proyecto de investigación financiado por 
el Ministerio de Educación y Ciencia Web Semántica y Sistemas de Información Documental (Referencia: HUM2004-03162/FILO). [Barcelona: UPF. Área de Biblioteconomía y Documentación. Dep. de Periodismo y de Comunicación Audiovisual, 2006, p. 56 ]

CODINA, Ll. et al (2010). "Cibermedios y Web 2.0: modelo de análisis resultados de aplicación". El profesional de la información .[en línea]. Vol. 19, núm. 1: p.35-44. [Consultado: 20 de abril de 2010]. Disponible en Internet: http://www.lluiscodina. com/periodismo20.pdf

DÍAZ NOCI, J. (2005). "Historia de los cibermedios en España". A: Cibermedios. El impacto de Internet en los medios de comunicación en España. Sevilla: Comunicación Social, Ediciones y Publicaciones. p. 21-38.

IGLESIAS, M. (2008). "Les edicions locals de VilaWeb al País Valencià" A: Comunicación local y nuevos formatos periodísticos en Internet: cibermedios, confidenciales y weblogs. Valencia: Servei Editorial de la Universitat de València. p.153168.

LÓPEZ, C.; PASTOR, J.A. (2010). Actualización del modelo de portal periodístico de prensa española. Anales de Documentación [en línea]. Vol. 13, p. 177-184. Universidad de Murcia. [Consultado: 28 de julio de 2010]. Disponible en Internet: http://revistas.um.es/analesdoc/article/view/107141

LÓPEZ, X. et al (2005). "Tipología de los Cibermedios". A: Cibermedios. El impacto de Internet en los medios de comunicación en España. Sevilla: Comunicación Social, Ediciones y Publicaciones. p.46.

MASIP, P. (2008). "El ciberperiodismo en Catalunya: apuntes sobre el estado de la cuestión". A: Comunicación local y nuevos formatos periodísticos en Internet: cibermedios, confidenciales y weblogs. Valencia: Server de Publicacions de la Universitat de València. p. 35-46.

MESO, K.; LÓPEZ, G.; ALONSO, J.(2007).’Métodos de catalogación y tipología de cibermedios".Ponencia presentada en el I coloquio Internacional Brasil-Espanha sobre Cibermedios. Faculdade de Comunicaçao de Universidade Federal da Bahia 3-7. [en línea]. [Consultado: mayo de 2010]. Disponible en: http://www. apudacta.eu/wp-content/archivo/meso-lopez-alonso-metodos-catalogacion-y-tipologia-medios.pdf

OFICINA DE JUSTIFICACIÓN DE LA DIFUSIÓN (OJD) (1997-2010). [en línea]. [Consultado: marzo y abril de 2010]. Disponible en Internet: http://www.ojdinteractiva.es/

ORIHUELA, J.L. (2005). “Tipología de los cibermedios”.A: Cibermedios. El impacto de Internet en los medios de comunicación en España. Sevilla: Comunicación Social, Ediciones y Publicaciones. p. 39-82.

PALACIOS, M.; DÍAZ, N. (2009). Online Journalism: research methods. A multidisciplinary approach in comparative perspective. Bilbao: Servicio Editorial de la Universidad del País Vasco.

PARRA, D. (2008). "El diseño ciberperiodístico español: análisis de los casos de VilaWeb y ADN". Enl@ce; Revista Venezolana de Información, Tecnología y Conocimiento. [en línea]. Vol. 5. Núm, 3: p. 91-107 [Consultado: 23 de marzo 
de 2010]. Disponible en Internet: http://dialnet.unirioja.es/servlet/fichero_articulo? codigo $=2746214 \&$ orden $=0$

PARTAL, v. (2007). Periodismo Quàntic. Fent periodisme a Internet. L'experiència dels primers deu anys de VilaWeb. Illes Balears: Universitat de les Illes Balears. Server de Publicacions i Intercanvi Científic. ${ }^{\mathrm{a}}$ ed. ISBN 978-84-7632-994-8

RODRÍGUEZ, R.; PEDRAZA-JIMÉNEZ, R.(2009); "Prensa Digital y Web 2.0". Hipertext.net. ISNN 1695-5498. Núm, 7. [Consultado: 24 de junio de 2010]. Disponible en Internet: http://www.hipertext.net/web/pag297.htm

SALAVERRIA, R.; SÁBADA, CH. (2003): Towards New Media Paradigms. Content, Producers, Organisations and Audiences II COST A20 Internacional Conference Proceedings. Pamplona: Eunate. ISBN 84-7768-150-3

SALAVERRIA, R. (2005). Cibermedios. El impacto de Internet en los medios de comunicación en España. $1^{\mathrm{a}}$ ed. Sevilla: Comunicación Social Ediciones y Publicaciones.

\section{Los autores}

Francisco Hernández Soriano. Licenciado en Administración de Empresas por la Universidad de Lleida (UdL) y doctorando en el programa "La construcción Europea" del departamento de Filología Catalana y Comunicación.

Eduard Cristóbal-Fransi. Doctor en Economía por la Universidad de Lleida (UdL). Profesor del área de comercialización e investigación de mercados. Director del departamento de Administración de Empresas en la UdL. Su investigación está centrada en el comercio electrónico, implantación de las TIC y la evaluación de la calidad percibida. Ha participado en congresos nacionales e internacionales y publicado en revistas como International Journal of Market Research, International Journal of Marketing Studies, International Journal of Business and Management, International Journal of Services Technology and Management and Managing Service Quality, y ha colaborado en diferentes libros como "E-Commerce and V-Business: Digital Enterprise in the Twenty-First Century" o "Social E-Enterprise: Value Creation through ICT" entre otros. 